\title{
Correction to: Keratin 7 expression in hepatic cholestatic diseases
}

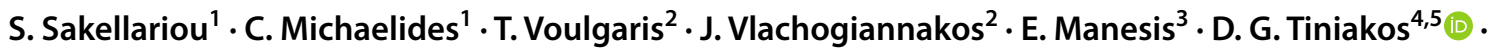 \\ I. Delladetsima ${ }^{1}$
}

Published online: 31 August 2021

(c) Springer-Verlag GmbH Germany, part of Springer Nature 2021

\section{Correction to: Virchows Archiv \\ https://doi.org/10.1007/s00428-021-03152-z}

In the original published version of this article, the presented Table 1 has incorrect presentation of data. The corrected table is shown as follows:

The original article has been corrected.

Publisher's Note Springer Nature remains neutral with regard to jurisdictional claims in published maps and institutional affiliations.

The original article can be found online at https://doi.org/10.1007/ s00428-021-03152-z.

D. G. Tiniakos

dina.tiniakos@newcastle.ac.uk

1 1st Department of Pathology, Medical School, Laiko General Hospital, National and Kapodistrian University of Athens, 75Mikras Asias str, 11527 Athens, Greece

2 Academic Department of Gastroenterology and Hepatology, Laiko General Hospital, National and Kapodistrian University of Athens, 17 Agiou Thoma str, 11527 Athens, Greece

3 Liver Unit, Euroclinic, 7-9 Athanasiadou Str., 11521 Athens, Greece

4 Department of Pathology, Aretaieion Hospital, National and Kapodistrian University of Athens, 76 Vasilissis Sofias Ave, 11528 Athens, Greece

5 Faculty of Medical Sciences, Translational \& Clinical Research Institute, Newcastle University, Framlington Place, Newcastle upon Tyne NE2 4HH, UK 
Table 1 Hepatocellular keratin 7 expression in all diagnostic categories and subgroups

\begin{tabular}{|c|c|c|c|c|c|c|}
\hline & \multicolumn{6}{|c|}{ K7 hepatocellular expression } \\
\hline & \multicolumn{2}{|l|}{ All zones } & \multicolumn{2}{|l|}{ Zone 1} & \multicolumn{2}{|l|}{ Zones $2 \& 3$} \\
\hline & $\begin{array}{c}\text { Score } \geq 1 \\
\mathrm{n}(\%)\end{array}$ & $\begin{array}{c}\text { Score } \geq 2 \\
\text { n }(\%)\end{array}$ & $\begin{array}{c}\text { Score } \geq 1 \\
\mathrm{n}(\%)\end{array}$ & $\begin{array}{c}\text { Score } \geq 2 \\
\mathrm{n}(\%)\end{array}$ & $\begin{array}{c}\text { Score } \geq 1 \\
\mathrm{n}(\%)\end{array}$ & $\begin{array}{c}\text { Score } \geq 2 \\
\mathrm{n}(\%)\end{array}$ \\
\hline All patients $(\mathrm{n}=92)$ & $81(87)$ & $55(60)$ & $79(86)$ & $54(60)$ & $67(73)$ & $10(32.6)$ \\
\hline Acute hepatitis $(n=20)$ & $16(80)$ & $5(25)$ & $15(75)$ & $4(20)$ & $11(55)$ & $5(25)$ \\
\hline Pure/mixed cholestasis $(n=16)$ & $12(75)$ & $6(37.5)$ & $11(68.8)$ & $6(37.5)$ & $12(75)$ & $3(18)$ \\
\hline iBDO $(n=48)$ & $46(96)$ & $38(79)$ & $46(96)$ & $39(79)$ & $38(79)$ & $17(35.4)$ \\
\hline $\operatorname{PBC}(n=35)$ & $34(97.1)$ & $26(74.35)$ & $34(97)$ & $26(74.3)$ & $26(74.3)$ & $10(28.6)$ \\
\hline $\operatorname{PSC}(n=10)$ & $10(100)$ & $10(100)$ & $10(100)$ & $10(100)$ & $10(100)$ & $5(50)$ \\
\hline $\operatorname{VBDS}(\mathrm{n}=3)$ & $2(66.7)$ & $2(66.7)$ & $2(66.7)$ & $2(66.7)$ & $2(66.7)$ & $2(66.7)$ \\
\hline $\operatorname{cBDO}(n=8)$ & $7(87.5)$ & $6(75)$ & $7(87.5)$ & $6(75)$ & $6(75)$ & $5(62.5)$ \\
\hline
\end{tabular}

"All zones" scores were calculated using the highest score in each case; K7 keratin 7, n number of cases, Z acinar zone, iBDO incomplete Bile Duct Obstruction/Destruction, PBC Primary Biliary Cholangitis, PSC Primary Sclerosing Cholangitis, VBDS Vanishing Bile Duct Syndrome, cBDO complete large Bile Duct Obstruction. 